\title{
A Systematic Review on the Effect of HIV Infection on the Pharmacokinetics of First-Line Tuberculosis Drugs
}

\author{
Alper Daskapan ${ }^{1} \cdot$ Lusiana R. Idrus $^{2} \cdot$ Maarten J. Postma $^{2,3} \cdot$ Bob Wilffert $^{1,2} \cdot$ Jos G. W. Kosterink $^{1,2} \cdot$ Ymkje Stienstra $^{4}$. \\ Daniel J. Touw ${ }^{1,5}$. Aase B. Andersen ${ }^{6}$. Adrie Bekker ${ }^{7}$. Paolo Denti ${ }^{8}$. Agibothu K. Hemanth Kumar ${ }^{9}$. \\ Kidola Jeremiah $^{10}$. Awewura Kwara ${ }^{11}$ • Helen Mcllleron ${ }^{8}$. Graeme Meintjes ${ }^{12}$. Joep J. van Oosterhout ${ }^{13,14}$. \\ Geetha Ramachandran ${ }^{9} \cdot$ Neesha Rockwood $^{12,15} \cdot$ Robert J. Wilkinson ${ }^{12,15,16} \cdot$ Tjip S. van der Werf $^{4}$. \\ Jan-Willem C. Alffenaar ${ }^{1}$
}

Published online: 8 November 2018

(c) The Author(s) 2018

\begin{abstract}
Introduction Contrasting findings have been published regarding the effect of human immunodeficiency virus (HIV) on tuberculosis (TB) drug pharmacokinetics (PK).

Objectives The aim of this systematic review was to investigate the effect of HIV infection on the PK of the first-line TB drugs (FLDs) rifampicin, isoniazid, pyrazinamide and ethambutol by assessing all published literature.

Methods Searches were performed in MEDLINE (through PubMed) and EMBASE to find original studies evaluating the effect of HIV infection on the PK of FLDs. The included studies were assessed for bias and clinical relevance. PK data were extracted to provide insight into the difference of FLD PK between HIV-positive and HIV-negative TB patients. This systematic review was conducted in accordance with the Preferred Reporting Items for Systematic Reviews and Meta-Analyses statement and its protocol was registered at PROSPERO (registration number CRD42017067250).

Results Overall, 27 studies were eligible for inclusion. The available studies provide a heterogeneous dataset from which consistent results could not be obtained. In both HIV-positive and HIV-negative TB groups, rifampicin (13 of 15) and ethambutol (4 of 8 ) peak concentration $\left(C_{\max }\right)$ often did not achieve the minimum reference values. More than half of the studies (11 of 20) that included both HIV-positive and HIV-negative TB groups showed statistically significantly altered FLD area under the concentration-time curve and/or $C_{\max }$ for at least one FLD.

Conclusions HIV infection may be one of several factors that reduce FLD exposure. We could not make general recommendations with respect to the role of dosing. There is a need for consistent and homogeneous studies to be conducted.
\end{abstract}

Jan-Willem C. Alffenaar

j.w.c.alffenaar@umcg.nl

Extended author information available on the last page of the article

\section{Key Points}

The available studies provide a heterogeneous dataset, and this study exposes the current knowledge gaps regarding the effect of human immunodeficiency virus (HIV) infection on the pharmacokinetics (PK) of firstline tuberculosis drugs (FLDs).

There is a need for a consistent and homogeneous approach to studies, and for a uniform quality assessment tool for PK studies.

Taking clinical relevance into account, we postulate that HIV infection may increase the risk for low FLD exposure, with potential detrimental consequences for treatment outcomes. 


\section{Introduction}

Tuberculosis (TB) is an infectious disease caused by the organism Mycobacterium tuberculosis. Despite concerted efforts, TB has remained a major global health problem [1]. With an estimated 1.8 million TB deaths in 2015, including 0.4 million TB-related deaths among human immunodeficiency virus (HIV) infected persons, TB is a leading infectious killer worldwide [1]. Although improvements have been made in the prevention and treatment of HIV, 2.1 million new HIV infections worldwide were reported in 2015, resulting in a total of 36.7 million people living with HIV globally [2]. The risk of developing TB is 17- to 22-fold higher for people living with HIV, making HIV the most important predisposing factor for TB [3, 4]. TB and HIV are known to act synergistically on the decline of the host immune response, which is fatal if left untreated $[5,6]$.

The treatment of drug-susceptible TB consists of four first-line TB drugs (FLD): isoniazid (INH), rifampicin (RIF), pyrazinamide (PZA) and ethambutol (EMB) [7]. Due to the limited resources in regions with a high TB burden, the World Health Organization (WHO) advocates standardized treatment with generic, fixed-dose combination (FDC) formulation tablets for reasons of adherence, costs and logistics [7]. The recommended regimen consists of a 2-month intensive phase with all four FLDs, and a 4-month continuation phase with RIF and INH only [7]. Despite the utilization of weight-banded dosing, high pharmacokinetic (PK) variability has been reported for the FLDs in studies investigating the PK of these drugs [8-10]. The hollow-fibre infection model and murine model conducted with the four FLDs showed that their effectiveness is best reflected by the area under the concentration-time curve (AUC)/minimum inhibitory concentration (MIC) ratio [9, 11-13]. Notably, high PK variability and inadequate TB drug exposure are undesirable as high drug concentrations could lead to toxicity, while low drug exposure predisposes to prolonged treatment, treatment failure, relapse, and development of drug resistance [9, 14-17]. Several factors are known to cause interindividual PK variability, including body weight [18], sex [18, 19], pharmacogenomics $[20,21]$ and comorbid conditions such as diabetes mellitus [19].

Contrasting findings have been published regarding the effect of HIV on TB drug PK variability. Some studies showed reduced FLD exposure in HIV-infected patients [22-24], while others found no impact of HIV co-infection [25, 26]. TB drug concentrations are an important determinant of clinical response to treatment [27] and any potential negative effect of HIV co-infection on the PK of TB drugs is therefore crucial. Despite the WHO recommendation that all individuals living with HIV should be initiated on antiretroviral therapy (ART), resulting in high ART coverage of HIV-infected TB patients, the effect of HIV infection on the PK of FLDs remains relevant. The start of ART does not immediately improve the clinical and immunological condition of the patient, and the high bacterial burden at the start of TB treatment increases the risk of acquired drug resistance if plasma drug concentrations are affected by HIV co-infection.

In high endemic TB areas, drug shortages delay ART initiation and HIV suppression is not always achieved with the available antiretroviral drugs. The aim of this systematic review was to investigate the impact of HIV infection on the PK of RIF, INH, PZA and EMB.

\section{Methods}

This systematic review was conducted in accordance with the Preferred Reporting Items for Systematic Reviews and Meta-Analyses (PRISMA) statement [28]. The protocol was registered at PROSPERO (registration number CRD42017067250).

A specific clinical question was structured according to the population, intervention, comparison, outcome (PICO) approach. In this process, ' $\mathrm{P}$ ' represented HIV-positive patients with TB co-infection; 'I' represented treatment of drug-susceptible TB with RIF, INH, PZA and EMB; 'C' represented HIV-negative TB patients; and ' $\mathrm{O}$ ' represented the drug concentration of RIF, INH, PZA and EMB.

To retrieve relevant articles, a systematic electronic database search was performed in MEDLINE (through PubMed) and EMBASE on 11 June 2017, and an additional check for new published articles was conducted on 29 August 2018. The searches were assessed to find original studies evaluating the effect of HIV infection on the PK of RIF, INH, PZA and/or EMB. All published studies, without restriction on language and publication date, were eligible. Studies in adult and paediatric populations were included. In cases where healthy volunteers were included as a control group, the study was eligible for inclusion, provided that a group of HIV-infected patients without TB was included to assess the effect of HIV infection on the PK of the FLDs. Studies with HIV-positive patients receiving ART were also eligible for inclusion, provided that the effect of HIV infection on the PK of the FLD was assessed and reported. Studies conducted in HIV-positive TB patients without a comparator HIV-negative TB group were included in the systematic review but were not eligible for in-depth analysis. Reviews, letters, meeting and abstract posters, and correspondence were excluded, as were studies without PK data, drug interaction studies, and nonhuman studies. 
The search terms used were: (hiv[mesh] OR hiv infection[mesh] OR hiv[tiab] OR hiv infection[tiab]) AND (tuberculosis[mesh] OR tuberculosis[tiab] OR tb[tiab]) AND ((pharmacokinetics[mesh] AND antitubercular agents[mesh]) OR (pharmacokinetics[tiab] AND (antitubercular[tiab] OR "TB drugs" [tiab] OR antimycobacterial[tiab] OR "antituberculosis drugs"[tiab] OR isoniazid[tiab] OR rifampicin[tiab] OR rifampin[tiab] OR ethambutol[tiab] OR pyrazinamide[tiab])). The studies retrieved from both PubMed and EMBASE were pooled and duplicate articles were removed. First, we screened titles and abstracts for eligibility, and full-text articles were read by the first author $(\mathrm{AD})$ if the abstract was found to be eligible or in case of doubt. When the full-text article met all inclusion and exclusion criteria, it was included in the systematic review. Primary references of the included studies were checked and included if relevant. A second reviewer (LRI) conducted the article selection process independently and any discrepancies were resolved by discussion. In order to identify unpublished studies, the ClinicalTrials.gov website (http://clinicaltrials.gov) was searched.

One researcher (AD) first performed data extraction, using a pre-discussed structured form, and the second researcher (LRI) then independently checked the data extraction. Variables including age group (paediatric or adult), comparator group(s) and the HIV-positive group were noted for the included articles. Dose, AUC, peak drug concentration $\left(C_{\max }\right)$, half-life $\left(t_{1 / 2}\right)$, time to reach $C_{\max }\left(T_{\max }\right)$, volume of distribution $\left(V_{\mathrm{d}}\right)$ and clearance $(\mathrm{CL})$ were extracted from the included articles if available and were stratified by group. The data were extracted and noted per drug of interest (RIF, INH, PZA and EMB). Corresponding authors were contacted by electronic mail for additional data if relevant data were missing in the included studies. Finally, the possibility of pooling data from included studies was assessed based on the risk of bias assessment, PK calculation strategy and data presentation.

No validated tool for risk of bias assessment of PK studies was available. In the absence of such a tool, we assessed the risk of bias in a study by noting the presence or absence of essential components required for adequate interpretation of results of a PK study. This provided the opportunity to compare the included studies on risk of bias related to the methods and design. The following components were checked: total sample size, inclusion of both HIV-positive and HIV-negative TB groups, proportion of participants with $\mathrm{CD}^{+}<200$ cells $/ \mu \mathrm{L}$ or $\mathrm{CD} 4 \%<12$, proportion of HIVpositive participants receiving ART, presence of an absorption test, report of PK-altering morbidities (gastrointestinal, hepatic or renal), assessment of interacting comedication, calculation of the drug dosage per included group, report of directly observed therapy (DOT), number of plasma samples drawn per participant, description of specimen handling, use of validated analytical methods, method of AUC calculation, AUC calculation, stratification of data by HIV infection, and the number of participants who were lost to follow-up or died during the study period. Studies without a comparator group were only included in the narrative results and were excluded from further analysis. The combination of the number of plasma samples and the AUC calculation method (noncompartmental or model-based) was used to determine whether a study had a high or low risk of bias for the AUC calculation. Five or more plasma samples per patient, as well as utilization of a validated population PK model for all FLDs, were considered low risk.

In addition to a narrative synthesis of the results, the main results per study and the effect of HIV infection on AUC and/or $C_{\max }$ and additional PK parameters, if available, were displayed in a table. The data from patients at different months of treatment or at different dosing schemes were presented separately. When the AUC and/or $C_{\max }$ for both HIVpositive and HIV-negative TB groups were available, these results were plotted in histograms for each study, comparing the AUC and/or $C_{\max }$ between HIV groups. This provided the opportunity to demonstrate an overview of trends. The clinical relevance of our findings was assessed in accordance with European Medicines Agency (EMA) guidelines $[29,30]$. EMA guidelines including bioequivalence cut-off values of $<80 \%$ and $>125 \%$ were also used to estimate the clinical relevance of the reported statistically significant differences. Studies showing a statistically significant difference in AUC with an HIV-positive/HIV-negative ratio of $<80 \%$ or $>125 \%$ were considered clinically relevant. Only studies reporting data stratified by HIV status were eligible for this analysis.

\section{Results}

In total, 282 articles were retrieved from the searches in PubMed and EMBASE. Systematically assessing the retrieved articles resulted in 25 articles being eligible for inclusion. One additional article was a report of a preliminary analysis [31] of the study by Antwi et al. [32] and was therefore excluded. Two further articles were identified by reviewing the references of the first included articles [33, 34], resulting in a total of 27 articles being included in the current systematic review. No relevant unpublished studies were found on the ClinicalTrials.gov website investigating the effect of HIV infection on the PK of the FLDs. A flowchart of the selection process is presented in Fig. 1.

All included articles were screened for the presence or absence of essential components as a means of bias risk assessment. Twenty studies were conducted in adults and seven studies were conducted in children. Five studies only included an HIV-positive TB group, whereas a comparator 


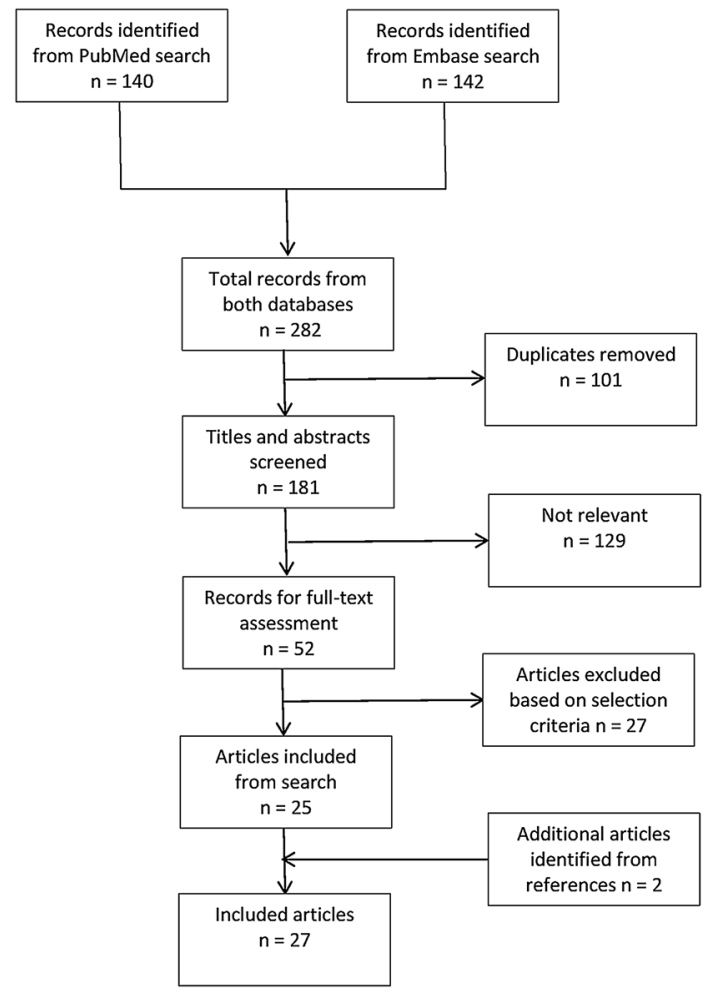

Fig. 1 Study search and selection process

HIV-negative TB group was lacking [34-38]; therefore these studies were excluded for further analysis. Twelve studies only included HIV-positive TB participants not receiving ART [22-24, 26, 32, 33, 39-44], in ten studies a proportion of HIV-positive participants were receiving ART [19, 34, 38, 45-51], and five studies did not provide information on ART use among HIV-positive TB patients [25, 35-37, 52]. In 11 studies, a limited number of fewer than five blood samples were drawn for determination of drug concentrations $[19,25$, 34, 36, 37, 40, 41, 43, 46, 47, 52]. Three studies included both an HIV-positive and an HIV-negative TB group but did not provide the AUC and $C_{\max }$ stratified by HIV status [40, 44, 46]. Two studies only reported $C_{\max }[19,52]$, while one study determined the percentage of RIF excreted in urine [23]. The assessment of risk of bias is presented in Table 1 .

Analysis of the extracted data showed that there was clinical, methodological and statistical heterogeneity among the included studies. The clinical heterogeneity consisted of diversity in outcomes, since outcomes were demonstrated as $\mathrm{AUC}_{4}, \mathrm{AUC}_{6}, \mathrm{AUC}_{8}, \mathrm{AUC}_{12}, \mathrm{AUC}_{24}$ and $\mathrm{AUC}_{\infty}$. The methodological diversity consisted of heterogeneity regarding sampling time points, number of samples collected, calculated AUC range, PK calculation methods and presentation of the results. As a result of the clinical and methodological heterogeneity, the data also showed high statistical heterogeneity as the main outcomes were inconsistent. As a result of the diversity, the data were too heterogeneous to allow pooling. The PK variability within and between studies was high for all four drugs when comparing the mean or median AUC and $C_{\max }$. The majority of studies presenting PK data reported AUC (16 of 27 studies) and $C_{\max }$ ( 21 of 27 studies). One study reported data on $V_{\mathrm{d}}$ [32], two reported data on CL $[32,46]$, eight reported data on $T_{\max }[22,26,32,33,36,37$, $47,53]$, and six reported data on $t_{1 / 2}[22,33,36,37,39,45]$.

\subsection{Rifampicin}

In total, 21 of the included articles assessed the effect of HIV infection on RIF PK. A narrative synthesis of the results is presented in Table 2. Three articles reported a statistically significantly reduced RIF AUC for the HIV-positive TB group compared with the HIV-negative TB group [22, $32,49]$. One article found that the HIV group had a statistically significantly lower RIF AUC value compared with healthy HIV-uninfected volunteers [33], while another study found the RIF AUC was statistically significantly higher in the HIV-positive TB group than in the HIV-negative TB group [26]. Five articles reported a statistically significant reduction of $C_{\max }$ in the HIV-positive TB group compared with the HIV-negative TB group [22, 32, 43, 49, 52], and one study showed a statistically significantly lower RIF $C_{\max }$ for the HIV group compared with healthy HIV-uninfected volunteers [33]. One study demonstrated that excretion of RIF was reduced by $27 \%$ and $34 \%$ in the HIV-positive group with diarrhoea and the HIV TB co-infected group without diarrhoea, respectively, compared with the HIV-uninfected TB group [23]. None of the included articles reported a statistically significant difference in $T_{\max }$ between the HIVnegative and HIV-positive TB groups. Histograms from studies comparing the HIV-negative and HIV-positive TB groups are plotted in Figs. 2a and 3a in regard to AUC and $C_{\text {max }}$, respectively.

\subsection{Isoniazid}

Twenty included articles assessed the effect of HIV on INH PK (Table 2), and none showed statistically significant differences in AUC between the HIV-negative and HIV-positive TB groups. Two studies, both conducted in India, reported a statistically significantly lower $C_{\max }$ in the HIV-positive TB group compared with the HIV-negative TB group [22, 49], and one study showed a shorter $T_{\max }$ for the HIV-positive TB group compared with the HIV-negative TB group [32]. In the one study that measured excretion of INH, a significant reduction of the excretion by $24 \%$ was found in the HIV-positive group with diarrhoea, and by $23 \%$ in the HIV-positive TB group without diarrhoea, compared with the HIV-uninfected TB group [23]. Histograms from studies comparing the HIV-negative and HIV-positive TB groups 


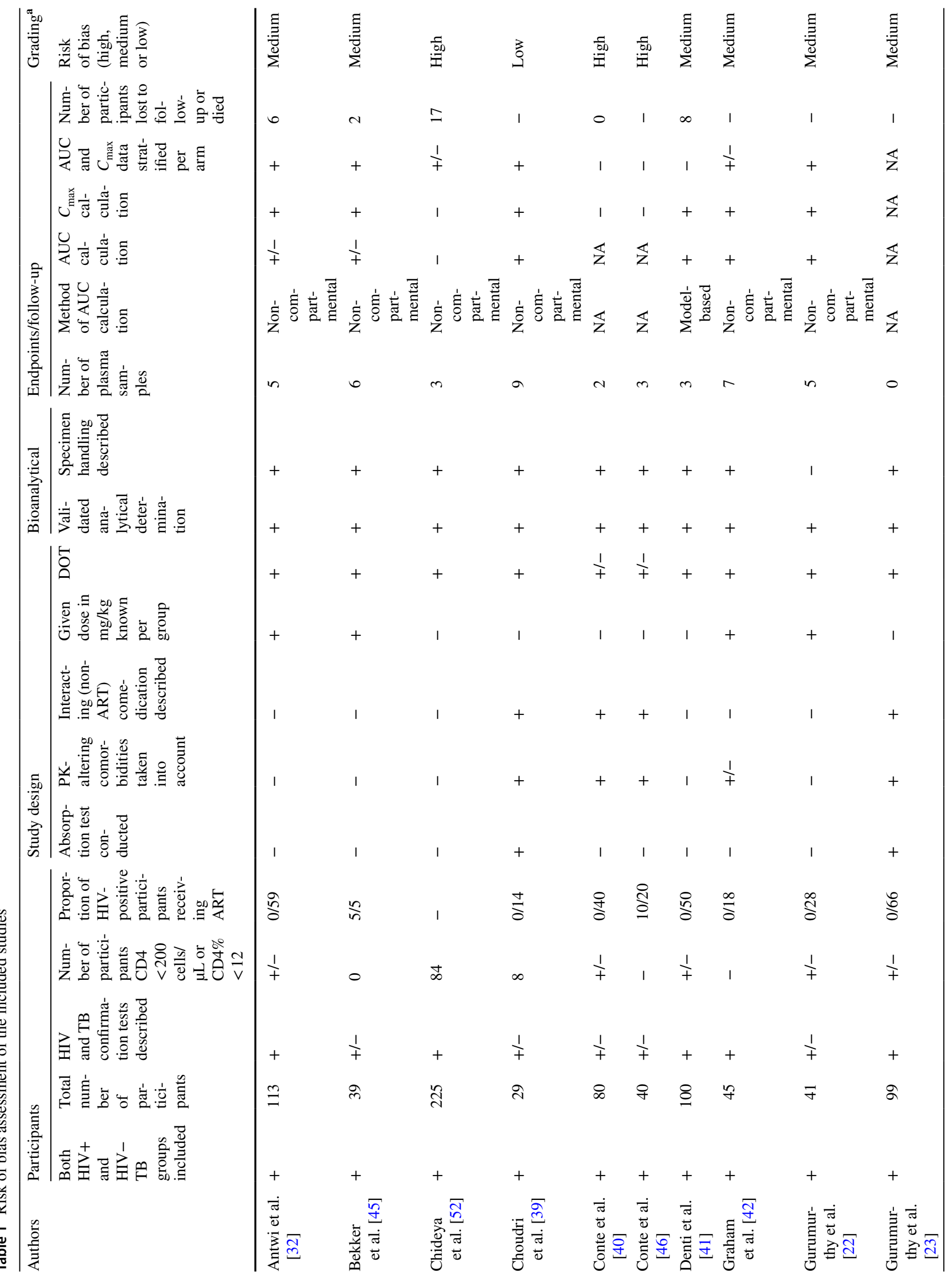




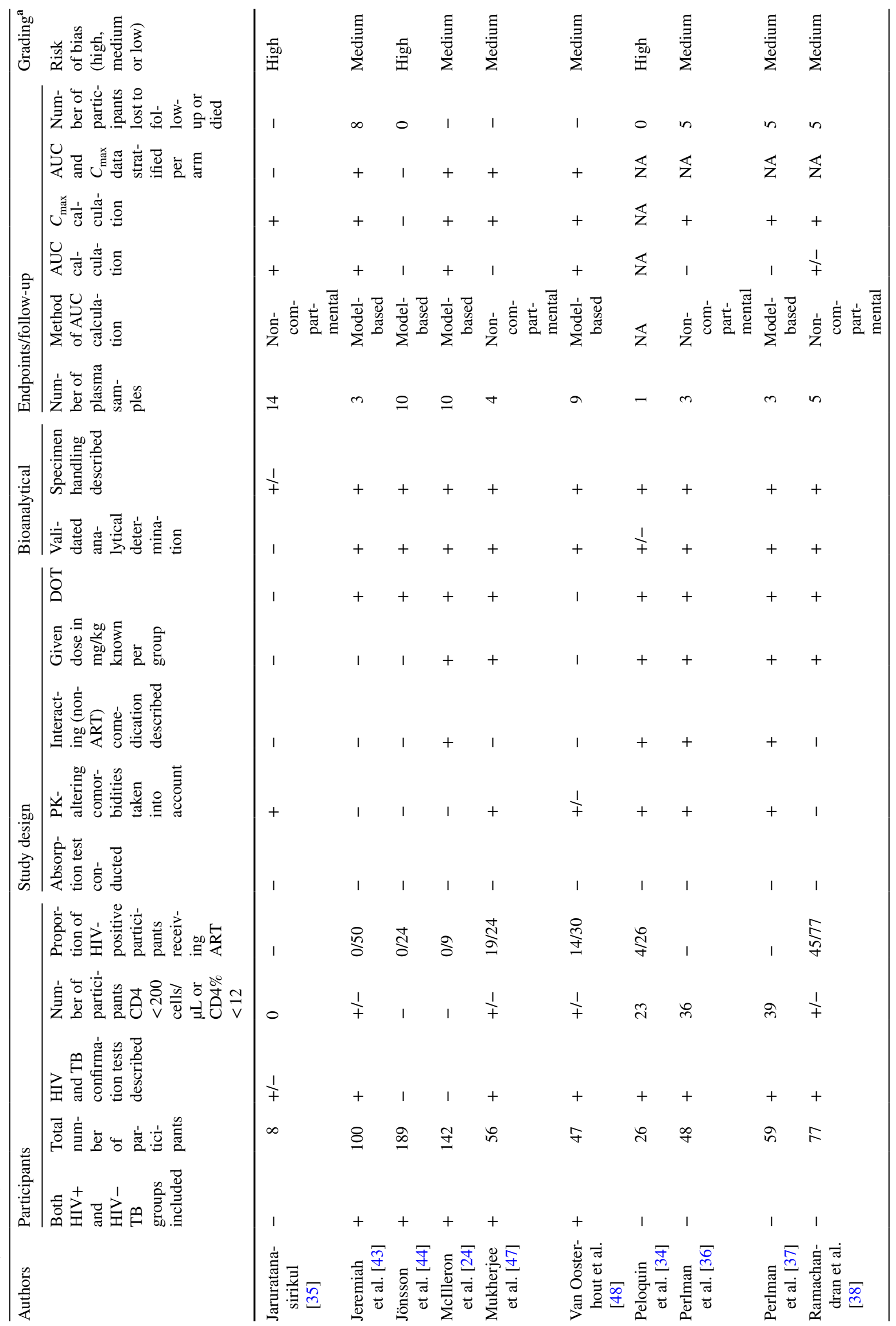




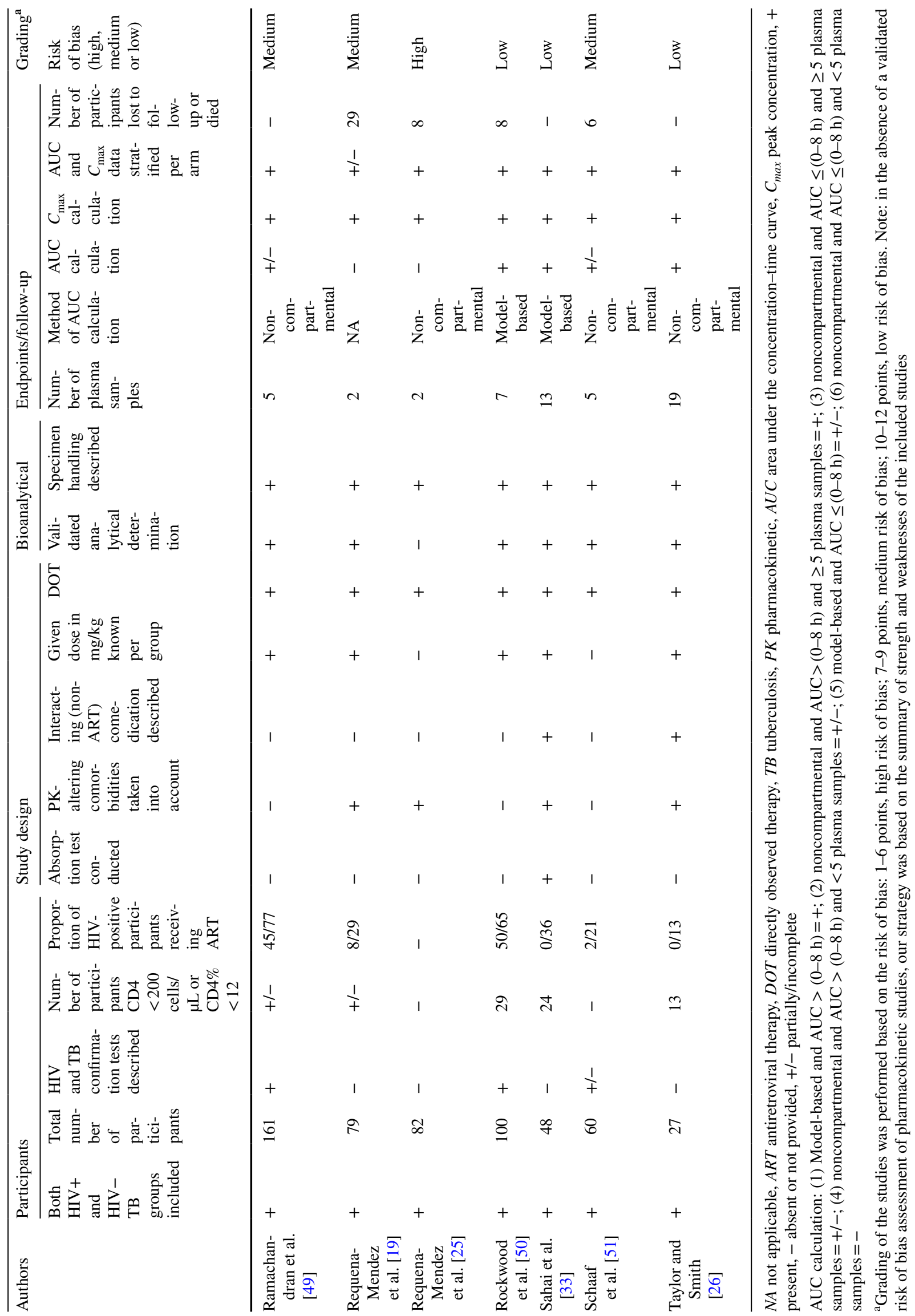




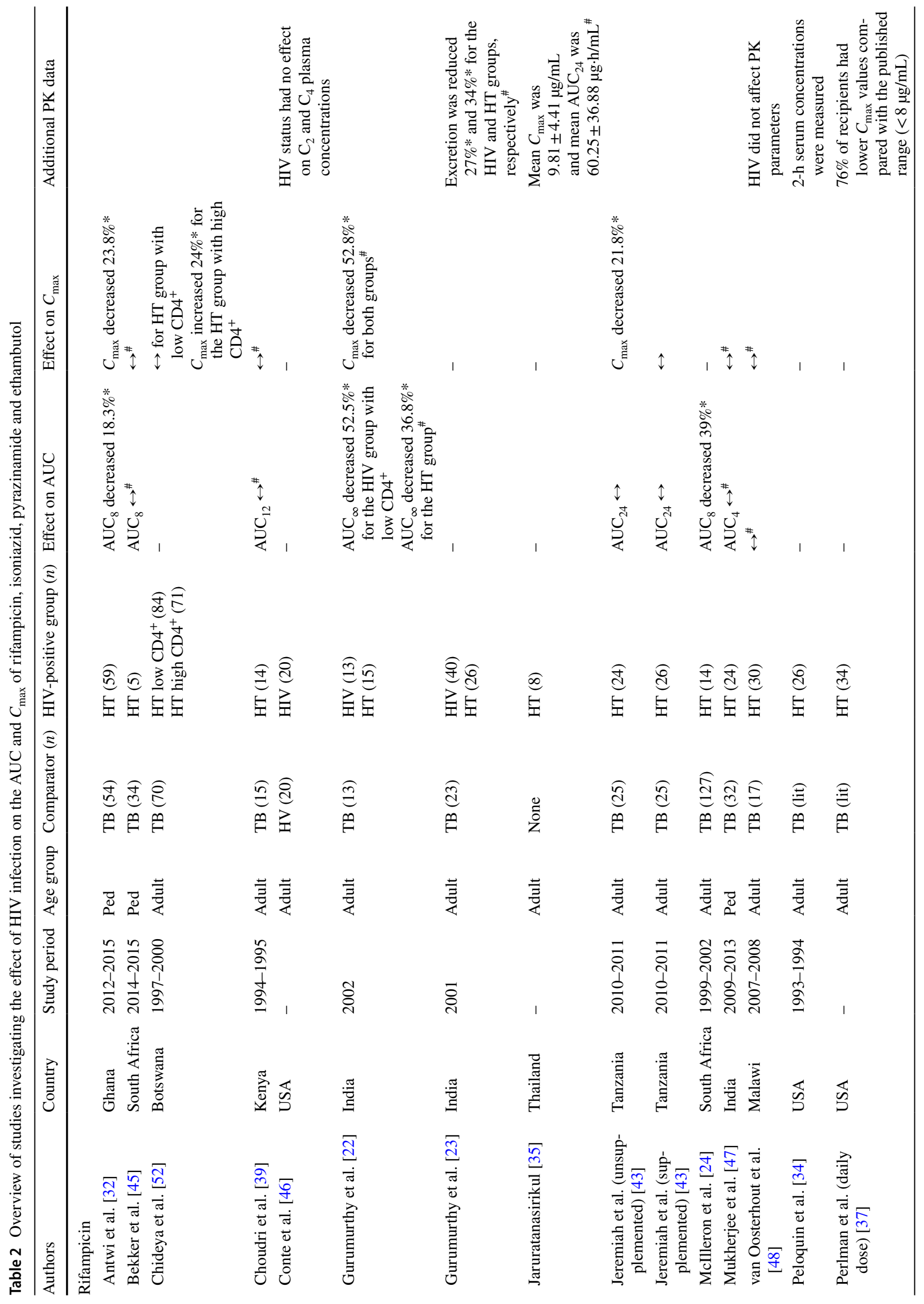




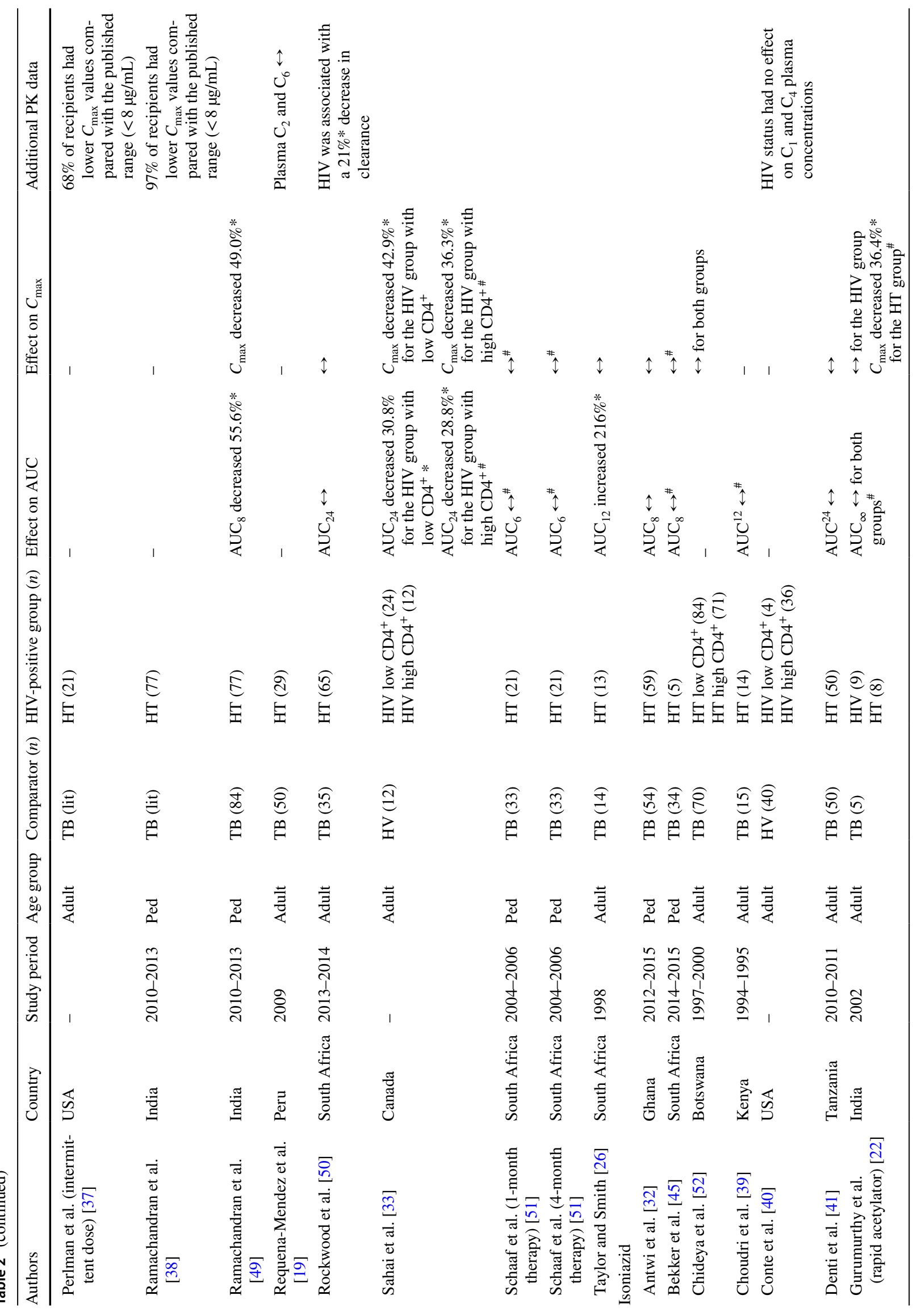




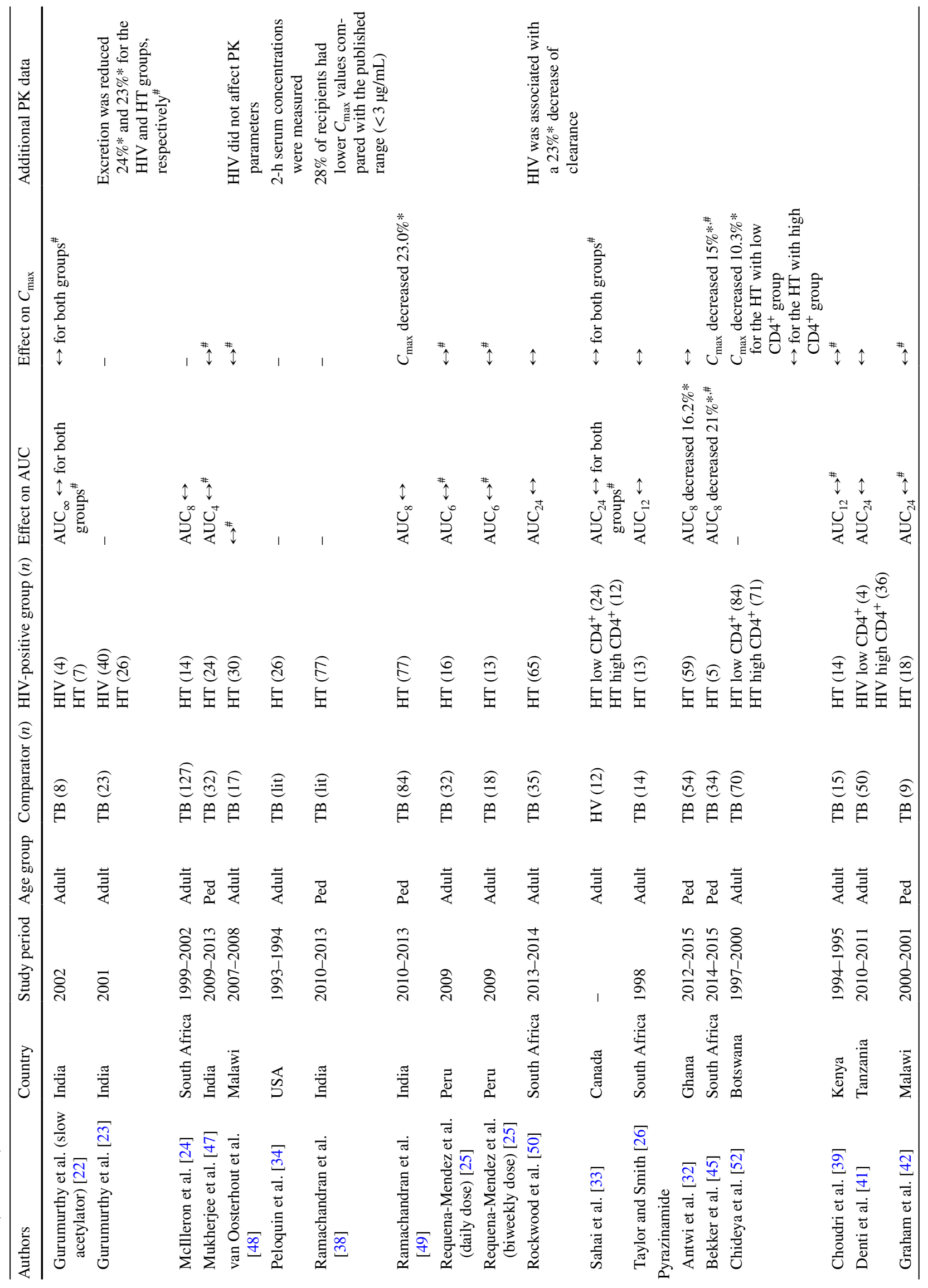



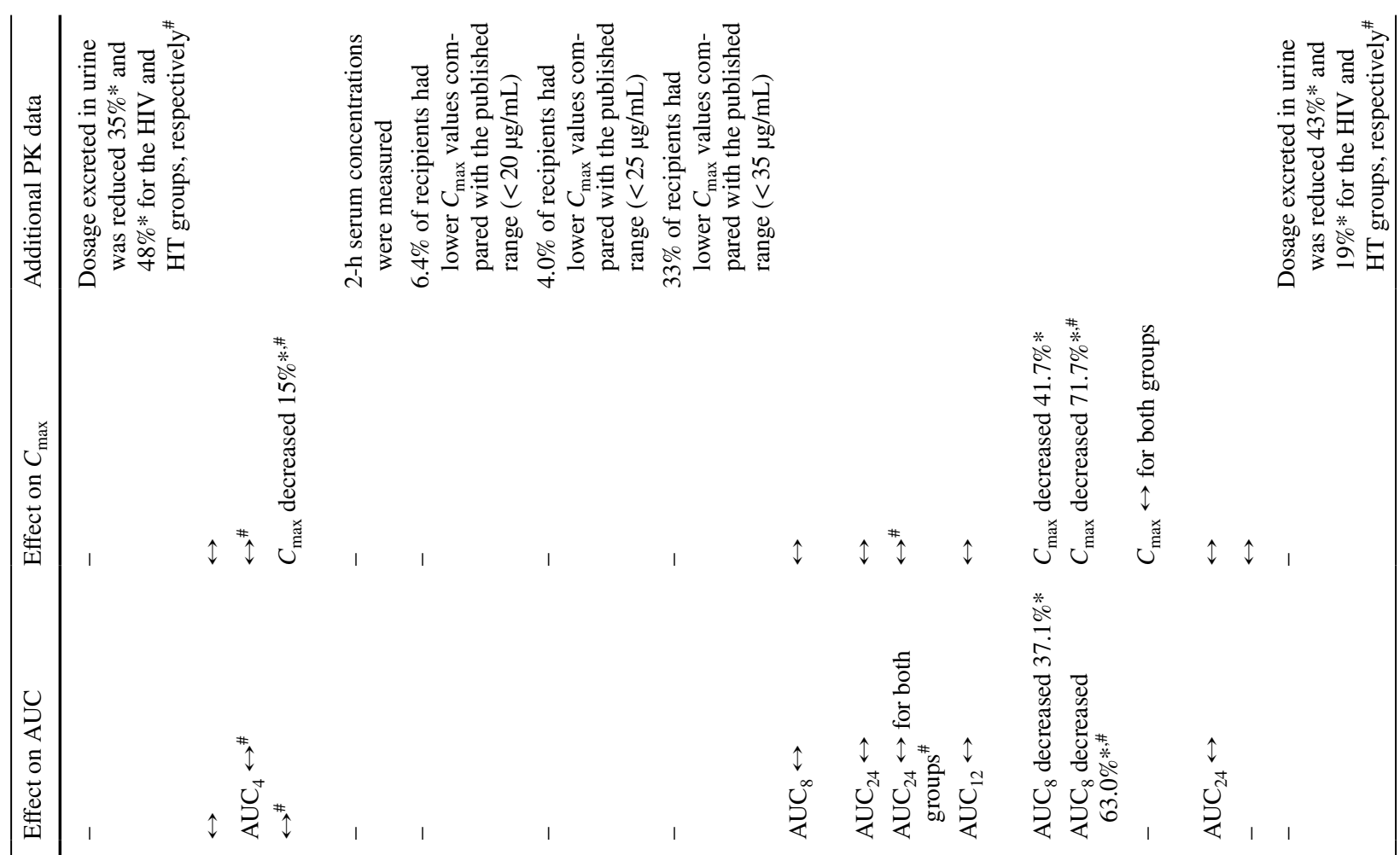

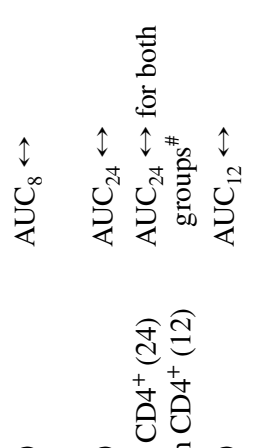

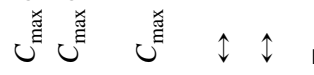

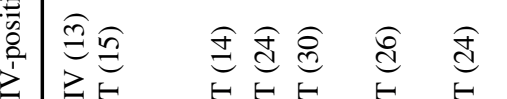

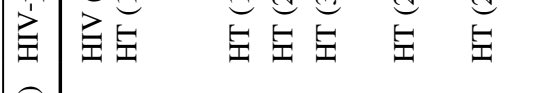

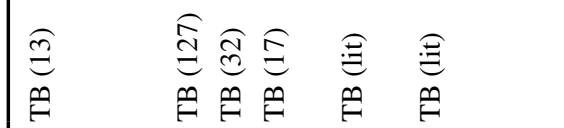

$\overparen{d} \quad E$

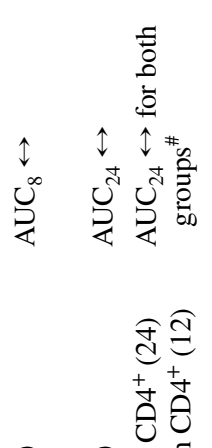

$\stackrel{*}{\stackrel{0}{*}}$

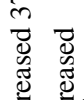

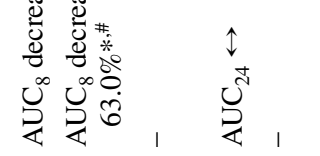

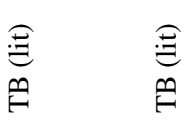

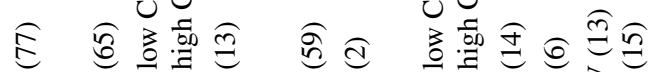

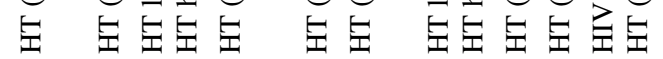

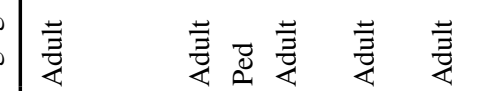

吾

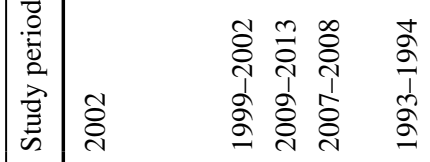

造

离

言重言

$\frac{\sqrt{5}}{\sum}$

芯

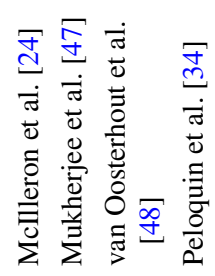

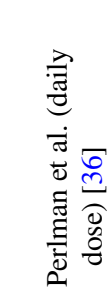

范

$\underset{g}{\stackrel{g}{g}}$

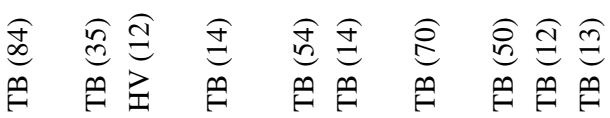

¿

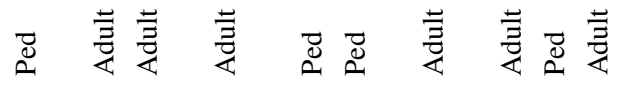

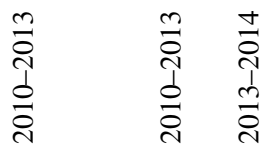

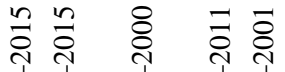

ำ

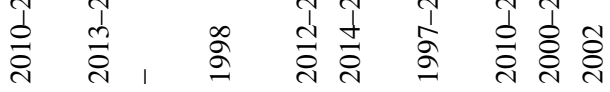

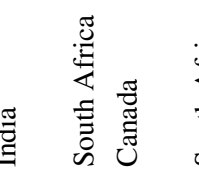

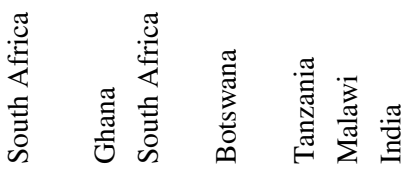

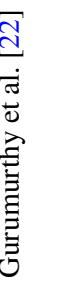

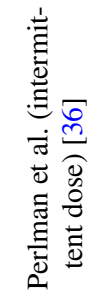

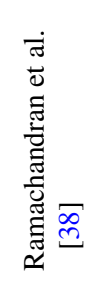

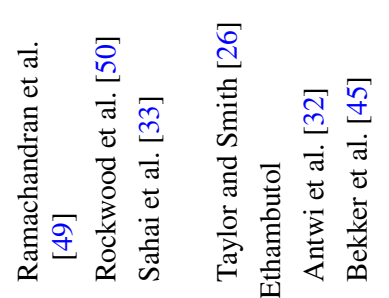

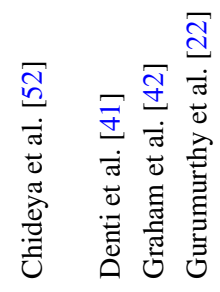




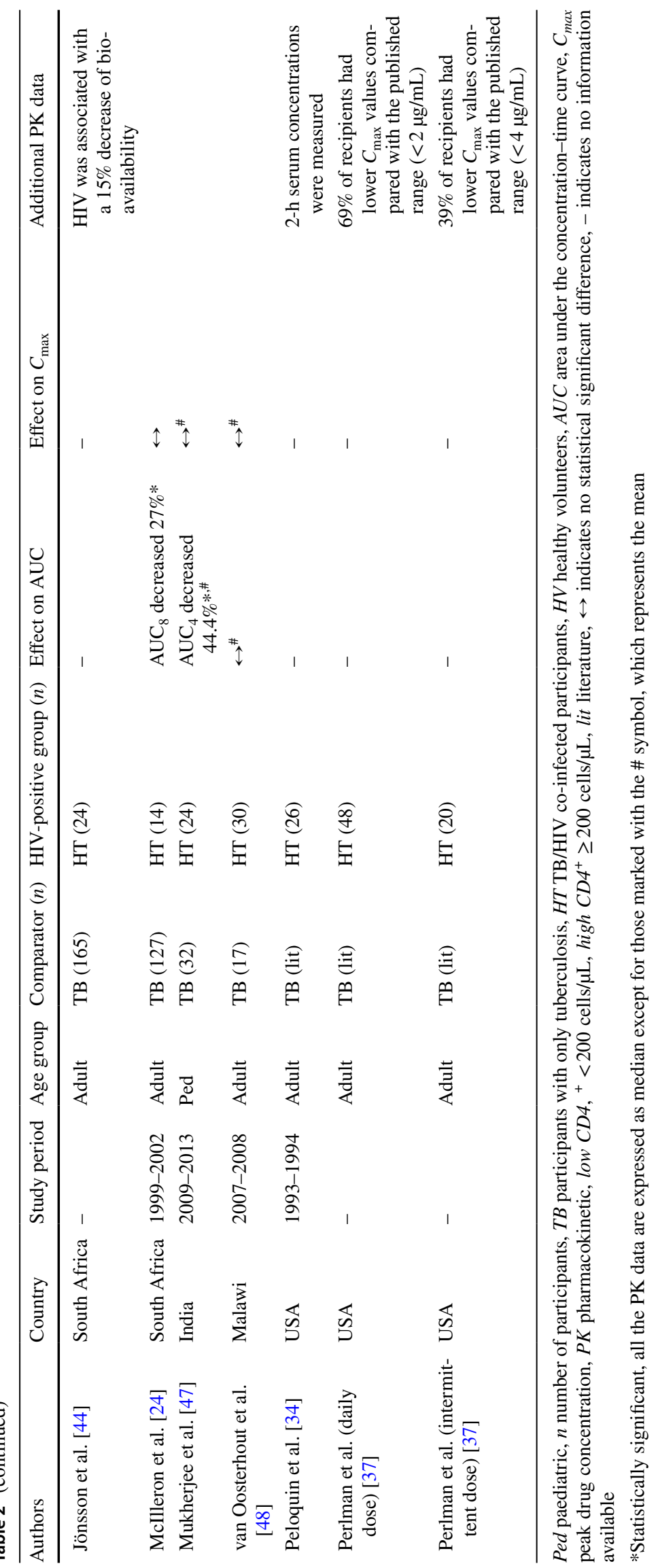



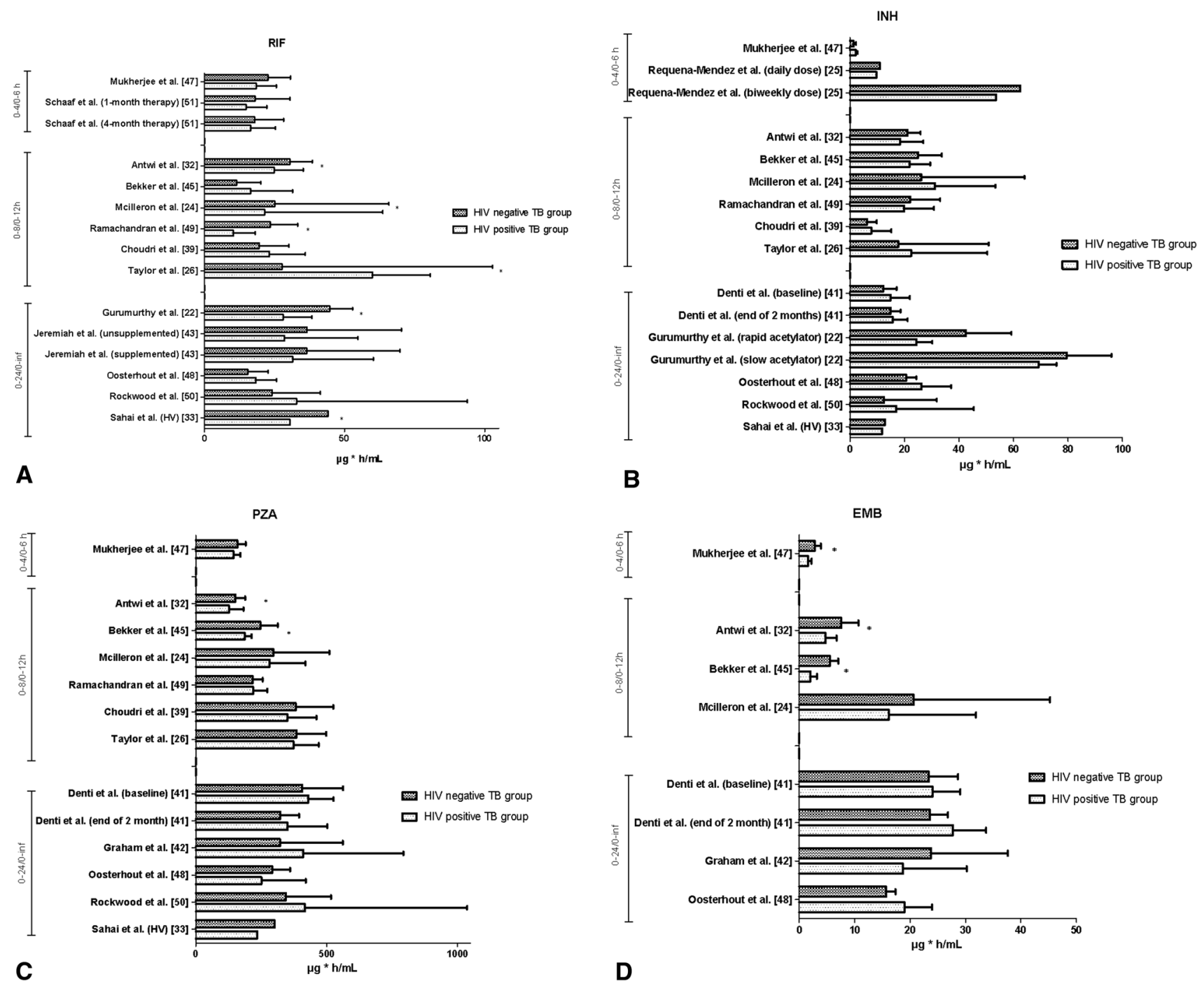

Fig. 2 Histograms of the mean or median area under the concentration-time curve for the HIV-negative and HIV-positive TB groups per study for a rifampicin, $\mathbf{b}$ isoniazid, $\mathbf{c}$ pyrazinamide and $\mathbf{d}$ ethambutol. Asterisk indicates statistical significance; 0-24/0-inf: $\mathrm{AUC}_{24}$ and $\mathrm{AUC}_{\infty} ; 0-8 / 0-12: \mathrm{AUC}_{8}$ and $\mathrm{AUC}_{12} ; 0-4 / 0-6 ; \mathrm{AUC}_{4}$ and $\mathrm{AUC}_{6}$. The

study by Sahai et al. [33] compared HIV-infected individuals without TB with healthy HIV-uninfected volunteers (healthy volunteers). INH isoniazid, $R I F$ rifampicin, $P Z A$ pyrazinamide, $E M B$ ethambutol, $T B$ tuberculosis

are plotted in Figs. $2 \mathrm{~b}$ and $3 \mathrm{~b}$ in regard to AUC and $C_{\max }$, respectively.

\subsection{Pyrazinamide}

Seventeen included articles assessed the effect of HIV on PZA PK (Table 2). Two articles found that the HIV-positive TB group had statistically significantly reduced AUC compared with the HIV-negative TB group [32, 45]. One article reported a statistically significant reduction of $C_{\max }$ in the HIV-positive TB group compared with the HIV-negative TB group [52], while another study showed a statistically significantly shorter $T_{\max }$ for the HIV-positive TB group compared with the HIV-negative TB group [32]. Histograms

from studies comparing the HIV-negative and HIV-positive TB groups are plotted in Figs. $2 \mathrm{c}$ and $3 \mathrm{c}$ in regard to AUC and $C_{\max }$, respectively

\subsection{Ethambutol}

Twelve included articles assessed the effect of HIV on EMB PK (Table 2). Three articles, all conducted in a paediatric population, showed that the HIV-positive TB group had statistically significantly reduced AUC compared with the HIVnegative TB group [32, 45, 47]. Two of these articles also reported a statistically significant reduction of $C_{\max }$ in the HIV-positive TB group compared with the HIV-negative TB group $[32,45]$. One study showed a statistically significant 


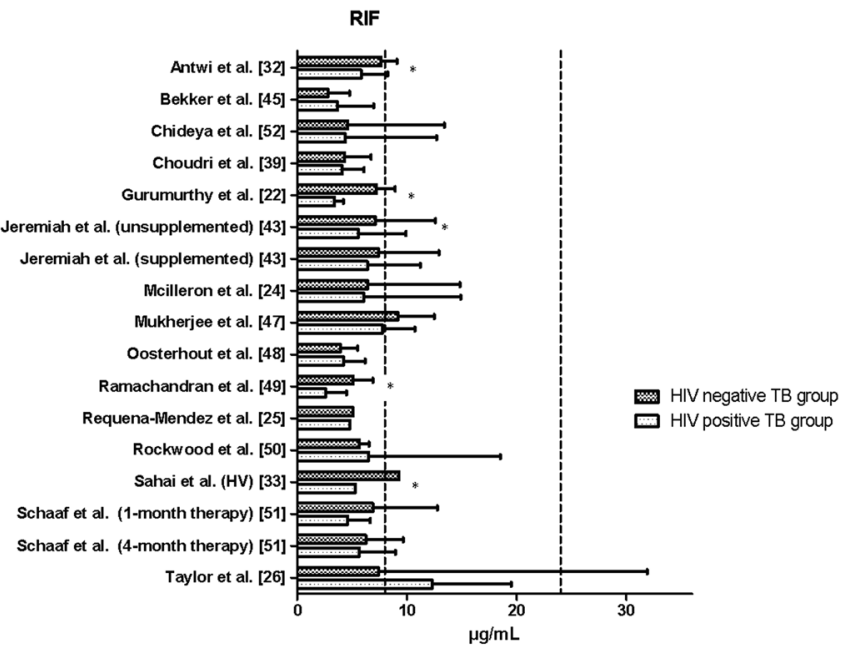

A

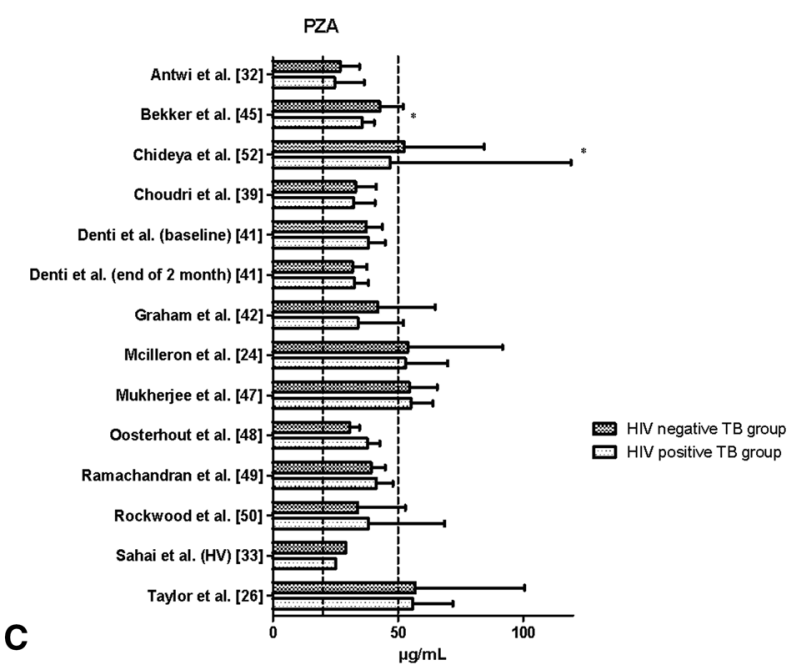

Fig. 3 Histograms of the mean or median peak drug concentration for the HIV-negative and HIV-positive TB groups per study for a rifampicin, $\mathbf{b}$ isoniazid, $\mathbf{c}$ pyrazinamide and $\mathbf{d}$ ethambutol. Asterisk indicates statistical significance. The dashed lines represent the generally cited reference ranges by Peloquin [27]: rifampicin 8-24 $\mu \mathrm{g}$ /

increase in $T_{\max }$ for the HIV-positive TB group compared with the HIV-negative TB group [47]. Histograms from studies comparing the HIV-negative and HIV-positive TB groups are plotted in Figs. $2 \mathrm{~d}$ and $3 \mathrm{~d}$ in regard to AUC and $C_{\max }$, respectively.

\subsection{Paediatrics}

Seven studies were conducted in paediatric populations [32, 38, 42, 45, 47, 49, 51]. One study lacked a comparator TB group [38], but the data were compared with the reference ranges reported by Alsultan and Peloquin [54]. These researchers concluded that the $C_{\max }$ of RIF, INH and PZA was subtherapeutic in $97 \%, 28 \%$ and $33 \%$ of children,

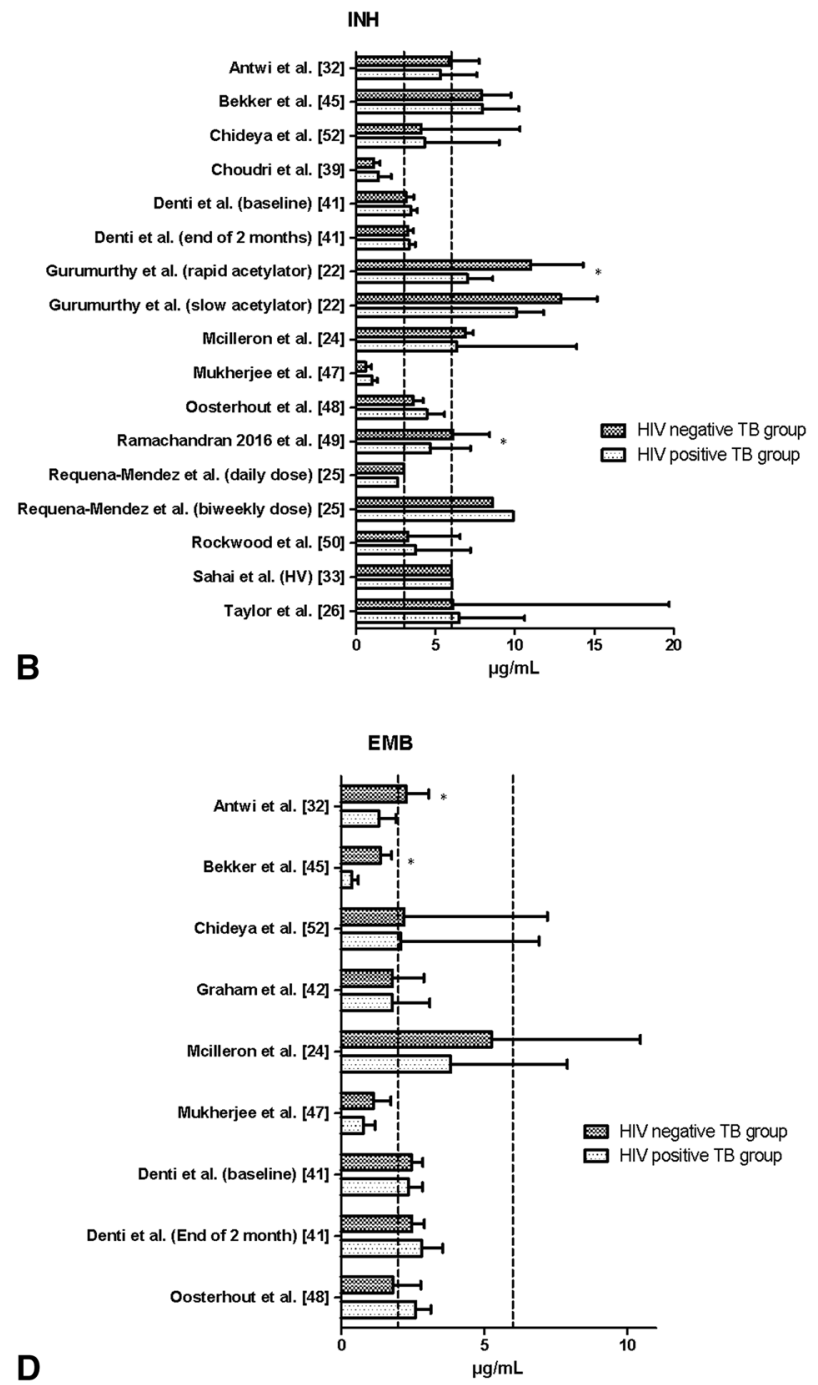

$\mathrm{mL}$; isoniazid 3-6 $\mu \mathrm{g} / \mathrm{mL}$; pyrazinamide $20-50 \mu \mathrm{g} / \mathrm{mL}$; ethambutol 2-6 $\mu \mathrm{g} / \mathrm{mL}$. The study by Sahai et al. [33] compared HIV-infected individuals without $\mathrm{TB}$ with healthy HIV-uninfected volunteers (healthy volunteers). INH isoniazid, RIF rifampicin, PZA pyrazinamide, $E M B$ ethambutol, $T B$ tuberculosis

respectively. Of the remaining six paediatric studies, four reported that HIV co-infection in children with TB adversely affects the AUC and/or $C_{\max }$ for at least one of the FLDs [32, 45, 47, 49], and two studies did not detect statistically significant differences between the groups $[42,51]$.

\subsection{Clinical Relevance}

The ratio in AUC between the HIV-positive and HIV-negative TB groups is shown in Fig. 4. Three of the four studies reporting a statistically significantly reduced RIF AUC for the HIV-positive TB group compared with the HIV-negative TB group were clinically relevantly reduced $(\leq 80 \%)[22,24$, 49]; the fourth study was not considered clinically relevantly 


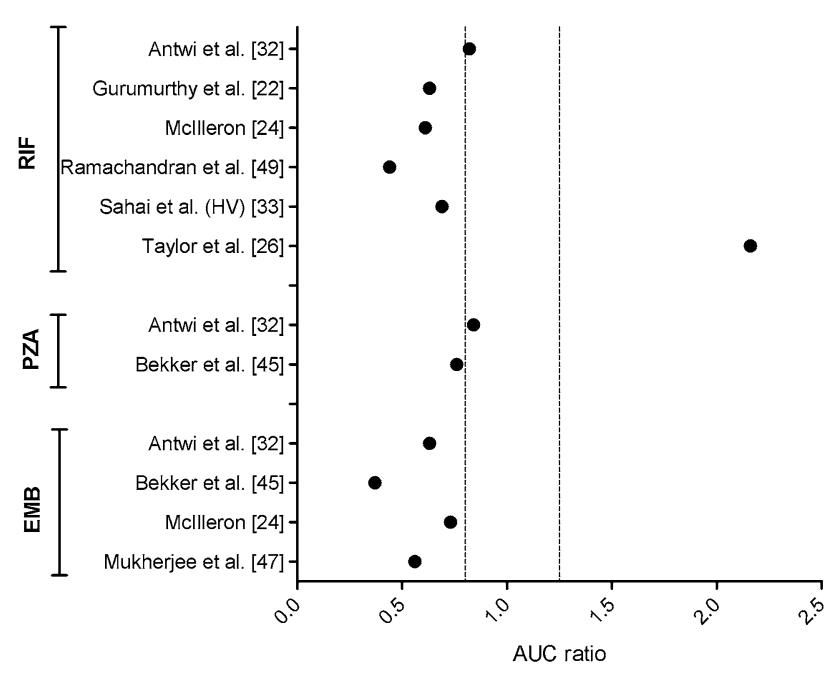

Fig. 4 Ratio between the AUCs of HIV-positive and HIV-negative TB patients for studies showing a statistically significant alteration in first-line TB drug AUCs, stratified per drug. The dashed lines represent the $80-125 \%(0.8-1.25)$ cut-off values for clinical relevance; all studies with a ratio outside this range were considered clinically relevant. $R I F$ rifampicin, $P Z A$ pyrazinamide, $E M B$ ethambutol, $T B$ tuberculosis, $A U C$ area under the concentration-time curve

reduced [32]. The decrease in RIF AUC reported in HIVpositive patients without TB compared with healthy volunteers [33] was considered clinically relevant. The one study reporting a statistically significant increase in RIF AUC in the HIV-positive TB group compared with the HIV-negative TB group [26] was also considered clinically relevant $(\geq 125 \%)$. Two studies demonstrated a statistically significantly reduced PZA AUC in the HIV-positive TB group compared with the HIV-negative TB group. One of these studies was considered borderline clinically relevant [45] and the other was not considered clinically relevant [32]. The results of all four studies showing a statistically significantly reduced EMB AUC in the HIV-positive TB group compared with the HIV-negative TB group were considered to be clinically relevant $[24,32,45,47]$.

\section{Discussion}

To our knowledge, this is the first systematic review investigating the effect of HIV infection on the PK of FLDs. We found that the published data were heterogeneous and no consistent results emerged from our literature review. We also found that for EMB, and in particular for RIF, both HIV-positive and HIV-negative TB groups often did not achieve the generally accepted threshold (or minimally acceptable) $C_{\max }$ reference range of $8 \mu \mathrm{g} / \mathrm{mL}$ for RIF and $2 \mu \mathrm{g} / \mathrm{mL}$ for EMB [27]. This phenomenon has already been observed in earlier studies, and research is currently being conducted investigating higher dosages of RIF [55, 56]. Although many studies showed a trend for lower AUC and/or $C_{\max }$ for at least one FLD in the HIV-positive TB group compared with the HIV-negative TB group, this did not always reach statistical significance. More than half of the studies (11 of 20) that included both HIV-positive and HIV-negative TB groups showed statistically significantly different AUC and/or $C_{\max }$ for at least one FLD [22, 24, $26,32,33,43,45,47-49,52]$. We focused on AUC and/ or $C_{\max }$ since most of the studies reported these as primary endpoints and they are the most relevant PK predictors of clinical outcomes, especially when combined with data on MIC [9, 11-13]. The majority of the articles focused on the PK of RIF and INH, which is justified by the fact that RIF and INH together are the backbone of drug-susceptible TB treatment.

The effect of HIV infection in TB patients on the PK of TB FLDs is an ongoing debate due to lack of consistent study results [54]. There may be several reasons to explain this inconsistency. First, several studies lacked a comparator group, making it difficult to adequately investigate the effect of HIV infection on the PK of the FLDs [34-38]. Instead, these studies compared with the widely cited reference ranges published by Peloquin et al. [27, 57] and Alsultan et al. $[54,58]$. However, these reference ranges are not age-, sex-, and weight-matched and often racial and regional differences are not taken into account. Studies have shown that female sex is a determinant of higher RIF, INH and PZA concentrations and lower EMB concentration [18, 24], and older age is a determinant of higher drug levels of all four FLDs [24, 58]. Another study reported that RIF exposure was significantly lower in people of African descent when adjusted for dose and genetic polymorphisms [59]. Although comparing PK data with published reference ranges provides a basic impression, patient characteristics differ highly between different populations, and conclusions from studies that compare PK findings with published reference ranges should therefore be regarded with caution.

Second, we postulate that the effect of HIV infection on the PK of the FLDs might often not have been detected due to a lack of power. Eleven of the studies that included both HIV-positive and HIV-negative TB groups showed statistically significantly that HIV infection adversely affects the PK (mainly AUC and/or $C_{\max }$ ) of at least one of the FLDs [22-24, 32, 33, 43-45, 47-49, 52]. Eight studies with both groups included did not detect a statistically significant difference between the two groups for all four FLDs [19, 25, 39-42, 46, 51], and one study even demonstrated a statistically significantly higher RIF AUC for the HIV-positive TB group [26]. Studies showing statistical differences in drug exposures to any of the FLDs had higher sample sizes and therefore more power compared with the studies that failed to detect such differences. 
The third potential contributor to the conflicting results published might be due to inadequate PK sampling and different PK calculation methods used. Studies unable to detect significant differences often had a lower number of collected blood samples for determination of drug concentrations. In addition to the varying numbers of collected blood samples, various different methods for AUC and $C_{\max }$ estimation were used. Some studies determined $C_{\max }$ by choosing the highest concentration among two or three blood draws [19, 25, $36,37]$. A more reliable method for estimation of $C_{\max }$ is fitting a population PK curve to the measured serum concentration-time data using Bayesian estimation [60]. Due to the varying number of blood samples drawn over a certain period of time, and the different methods used (model-based or noncompartmental) for the estimation of the AUC, the curves used to estimate AUCs in the included studies varied from 0 to $4 \mathrm{~h} \mathrm{[47]} \mathrm{to} 0$ to $24 \mathrm{~h}[48,50]$, thereby leading to potential loss of information. Collecting multiple blood samples over a longer period of time ensures the absorption, distribution, metabolism and elimination phases are adequately captured post-dose, which results in more accurate estimations of the AUC. Another approach is limited sampling strategies (LSS) or computational posteriori estimations using Bayesian methods [60-62]. In this systematic review, we therefore used the combination of the number of blood samples drawn, the use of PK modelling, and the implementation of validated bioanalytical methods for the assessment of risk of bias.

Another explanation for the contrasting results in the included studies is variation in the clinical severity of HIV infection, the degree of immunosuppression, and the use of ART. Several studies have demonstrated that the PK of the FLDs is more adversely altered in cases of more advanced stages of HIV [22, 33, 52]. In the studies that did not find lower drug exposures among HIV-positive TB patients compared with the HIV-negative TB group, the majority of co-infected patients had higher $\mathrm{CD} 4^{+}$cell counts and were receiving ART [41, 45, 48, 50], or data on HIV progression were lacking $[24,42]$. It is conceivable that successful ART mitigates the effect of HIV infection on TB drug PK parameters. In 10 studies, a proportion of the included HIV-positive participants was receiving ART [19, 34, 38, 45-51], while five studies did not provide information on ART use among HIV-positive TB patients [25, 35-37, 52]. The simultaneous use of FLD and ART can result in drug-drug interactions $[63,64]$ and can potentially lead to nonadherence.

Among the included studies, a high interindividual PK variability was found that was not merely attributable to HIV infection. We found that in the majority of studies, both HIV-positive and HIV-negative TB patients had an RIF $C_{\max }$ below the minimum reference range; the same applied for a proportion of the studies reporting EMB $C_{\max }$. This high variability involves the interplay of multiple factors, ranging from drug compounding to the distribution of the drug molecules at the site of infection. Drug formulation [24], pharmacogenomics [20, 21], racial and ethnic differences [20,59], sex [19, 24], body weight [18], advanced immunosuppression $[22,33]$, comorbid conditions such as diabetes mellitus $[19,23]$, comedication $[63,64]$ and nutritional status $[43,65]$ are the most investigated and salient factors. It is worth mentioning that a statistically significant reduction of FLD exposure in the HIV-positive TB groups does not necessarily have to be clinically relevant and that this should be explored in future studies that include treatment outcomes. In the absence of such studies at present, the cut-off values of the EMA guidelines $(<80 \%$ and $>125 \%)$ $[29,30]$ offer an alternative way to determine the clinical relevance of decreased or increased FLD exposures. Since these cut-off values are based on drug exposure, only studies reporting a statistically significant change in AUC could be included in the assessment. With the exception of the studies by Antwi et al. [32] and Taylor and Smith [26] in regard to RIF, and Antwi et al. [32] in regard to PZA AUC, all studies reporting a statistically significant alteration in FLD AUC were considered clinically relevantly reduced [22, 24, 26, 32, 33, 45, 47, 49]. Taking the risk of bias assessment (Table 1) into account in relation to the studies included in the systematic review, we postulate that in patients prone to low FLD exposure, HIV infection might even further reduce drug exposure [66], leading to poor treatment outcome [9]. While not a substitute for clinical judgement, therapeutic drug monitoring (TDM) could be a powerful tool for identifying patients with subtherapeutic FLD levels at risk of poor treatment outcomes $[62,67,68]$. TDM performed early during TB treatment in patients at risk of subtherapeutic FLD levels may improve treatment response and may also prevent toxicity $[68,69]$. For resource-limited settings, dried blood spot analysis combined with LSS or drug concentration measurements in saliva with thin-layer chromatography might provide a solution to address the problems of patients with a burden of blood draws, as well as costs [61, 70, 71].

A recent study by Hiruy et al. reported that HIV-negative children with TB are at risk of subtherapeutic concentrations for all FLDs [72]. Younger age has a considerable impact on TB drug exposure and should be considered in dosing recommendations. This has been attributed to children having a larger liver size and higher hepatic metabolic activity in proportion to body weight [53]. Our findings suggest that RIF and EMB exposures appear to be adversely affected in paediatric HIV-positive TB populations, even after administration of the revised WHO-recommended weight-based dosages. The clinical relevance of such reduced FLD exposures has to be urgently further investigated in paediatric populations.

A broad and comprehensive literature search was conducted systematically that allowed the identification of 
studies that included data on the effect of HIV infection on the PK of the FLDs. A strength of this systematic review is that it provides a good overview of the available literature and exposes current knowledge gaps. However, the systematic review also has some limitations. Despite the high disease burden, relatively few data were available and with variable quality, increasing the risk of bias. Furthermore, we chose to include all articles that included data on the effect of HIV infection on the PK of the FLDs, to prevent loss of information; therefore, studies lacking a comparator group and with participants receiving ART were included, potentially introducing bias. A more in-depth analysis was restricted to studies that had both an HIV-positive and HIVnegative TB group. Although no registered and unpublished studies were found in the database search, publication bias cannot be completely excluded. A recent study demonstrated that higher MIC values were associated with a greater risk of relapse than lower MIC values [73]. None of the studies included in this systematic review reported data on MIC. Lastly, the published data were too heterogeneous and were reported highly inconsistently, to allow pooling of the data. Due to methodological and statistical heterogeneity, subgroup analyses were also not appropriate.

\section{Conclusion}

Relatively few studies have been published investigating the effect of HIV infection on the PK of the FLDs. The available studies provide a heterogeneous dataset from which consistent results could not be obtained. Therefore, we could make no general recommendation with respect to dosing. There is a need for a consistent and homogeneous approach to studies and for a uniform quality assessment tool specifically for PK studies. Taking clinical relevance into account, we postulate that HIV infection may exacerbate a susceptibility to low FLD exposures, with potential detrimental consequences for treatment outcomes. This systematic review may inform further studies investigating the effect of HIV infection on the PK of the FLDs. A population PK analysis may provide a solution for the inability of pooling of the currently available data, as a population PK analysis can adjust for confounders. In addition, a prospective study with both an HIV-positive and HIV-negative TB group, including data on pharmacodynamics and treatment outcome, is needed to provide further insight into the highly complex PK of the FLDs.

\section{Compliance with ethical standards}

Funding No funding was received for this research.

Conflict of interest Alper Daskapan, Lusiana R. Idrus, Maarten J. Postma, Bob Wilffert, Jos G. W. Kosterink, Ymkje Stienstra, Daniel
J. Touw, Aase B. Andersen, Adrie Bekker, Paolo Denti, Agibothu K. Hemanth Kumar, Kidola Jeremiah, Awewura Kwara, Helen McIlleron, Graeme Meintjes, Joep J. van Oosterhout, Geetha Ramachandran, Neesha Rockwood, Robert J. Wilkinson, Tjip S. van der Werf and JanWillem C. Alffenaar declare that they have no conflicts of interest.

Open Access This article is distributed under the terms of the Creative Commons Attribution-NonCommercial 4.0 International License (http://creativecommons.org/licenses/by-nc/4.0/), which permits any noncommercial use, distribution, and reproduction in any medium, provided you give appropriate credit to the original author(s) and the source, provide a link to the Creative Commons license, and indicate if changes were made.

\section{References}

1. World Health Organization. Global tuberculosis report 2016; 2016. http://www.who.int/tb/publications/global_report/en/. Accessed 25 June 2017.

2. UNAIDS. Global AIDS update 2016; 2016. http://www.unaid s.org/en/resources/documents/2016/Global-AIDS-update-2016. Accessed 25 June 2017.

3. Getahun H, Gunneberg C, Granich R, Nunn P. HIV infectionassociated tuberculosis: the epidemiology and the response. Clin Infect Dis. 2010;50(Suppl 3):S201-7.

4. Selwyn PA, Hartel D, Lewis VA, Schoenbaum EE, Vermund SH, Klein RS, et al. A prospective study of the risk of tuberculosis among intravenous drug users with human immunodeficiency virus infection. N Engl J Med. 1989;320(9):545-50.

5. Modjarrad K, Vermund SH. Effect of treating co-infections on HIV-1 viral load: a systematic review. Lancet Infect Dis. 2010;10(7):455-63.

6. Whalen C, Horsburgh CR, Hom D, Lahart C, Simberkoff M, Ellner J. Accelerated course of human immunodeficiency virus infection after tuberculosis. Am J Respir Crit Care Med. 1995;151(1):129-35.

7. World Health Organization. Treatment of tuberculosis guidelines fourth edition; 2010. http://www.who.int/tb/publicatio ns/2010/9789241547833/en/. Accessed 25 June 2017.

8. Devaleenal DB, Ramachandran G, Swaminathan S. The challenges of pharmacokinetic variability of first-line anti-TB drugs. Expert Rev Clin Pharmacol. 2017;10(1):47-58.

9. Pasipanodya JG, McIlleron H, Burger A, Wash PA, Smith P, Gumbo T. Serum drug concentrations predictive of pulmonary tuberculosis outcomes. J Infect Dis. 2013;208(9):1464-73.

10. Reynolds J, Heysell SK. Understanding pharmacokinetics to improve tuberculosis treatment outcome. Expert Opin Drug Metab Toxicol. 2014;10(6):813-23.

11. Gumbo T, Louie A, Deziel MR, Liu W, Parsons LM, Salfinger M, et al. Concentration-dependent Mycobacterium tuberculosis killing and prevention of resistance by rifampin. Antimicrob Agents Chemother. 2007;51(11):3781-8.

12. Gumbo T, Louie A, Liu W, Brown D, Ambrose PG, Bhavnani SM, et al. Isoniazid bactericidal activity and resistance emergence: integrating pharmacodynamics and pharmacogenomics to predict efficacy in different ethnic populations. Antimicrob Agents Chemother. 2007;51(7):2329-36.

13. Jayaram R, Gaonkar S, Kaur P, Suresh BL, Mahesh BN, Jayashree $\mathrm{R}$, et al. Pharmacokinetics-pharmacodynamics of rifampin in an aerosol infection model of tuberculosis. Antimicrob Agents Chemother. 2003;47(7):2118-24.

14. Van't Boveneind-Vrubleuskaya N, Daskapan A, Kosterink JG, van der Werf TS, van den Hof S, Alffenaar JC. Predictors of 
prolonged TB treatment in a Dutch outpatient setting. PLoS One. 2016;11(11):e0166030.

15. Srivastava S, Pasipanodya JG, Meek C, Leff R, Gumbo T. Multidrug-resistant tuberculosis not due to noncompliance but to between-patient pharmacokinetic variability. J Infect Dis. 2011;204(12):1951-9.

16. Mehta JB, Shantaveerapa H, Byrd RP Jr, Morton SE, Fountain F, Roy TM. Utility of rifampin blood levels in the treatment and follow-up of active pulmonary tuberculosis in patients who were slow to respond to routine directly observed therapy. Chest. 2001;120(5):1520-4.

17. Weiner M, Benator D, Burman W, Peloquin CA, Khan A, Vernon A, Tuberculosis Trials Consortium, et al. Association between acquired rifamycin resistance and the pharmacokinetics of rifabutin and isoniazid among patients with HIV and tuberculosis. Clin Infect Dis. 2005;40(10):1481-91.

18. McIlleron H, Rustomjee R, Vahedi M, Mthiyane T, Denti P, Connolly $\mathrm{C}$, et al. Reduced antituberculosis drug concentrations in HIV-infected patients who are men or have low weight: implications for international dosing guidelines. Antimicrob Agents Chemother. 2012;56(6):3232-8.

19. Requena-Mendez A, Davies G, Ardrey A, Jave O, Lopez-Romero SL, Ward SA, et al. Pharmacokinetics of rifampin in Peruvian tuberculosis patients with and without comorbid diabetes or HIV. Antimicrob Agents Chemother. 2012;56(5):2357-63.

20. Chigutsa E, Visser ME, Swart EC, Denti P, Pushpakom S, Egan D, et al. The SLCO1B1 rs4149032 polymorphism is highly prevalent in South Africans and is associated with reduced rifampin concentrations: dosing implications. Antimicrob Agents Chemother. 2011;55(9):4122-7.

21. Parkin DP, Vandenplas S, Botha FJ, Vandenplas ML, Seifart HI, van Helden PD, et al. Trimodality of isoniazid elimination: phenotype and genotype in patients with tuberculosis. Am J Respir Crit Care Med. 1997;155(5):1717-22.

22. Gurumurthy P, Ramachandran G, Hemanth Kumar AK, Rajasekaran S, Padmapriyadarsini C, Swaminathan S, et al. Decreased bioavailability of rifampin and other antituberculosis drugs in patients with advanced human immunodeficiency virus disease. Antimicrob Agents Chemother. 2004;48(11):4473-5.

23. Gurumurthy P, Ramachandran G, Hemanth Kumar AK, Rajasekaran S, Padmapriyadarsini C, Swaminathan S, et al. Malabsorption of rifampin and isoniazid in HIV-infected patients with and without tuberculosis. Clin Infect Dis. 2004;38(2):280-3.

24. McIlleron H, Wash P, Burger A, Norman J, Folb PI, Smith P. Determinants of rifampin, isoniazid, pyrazinamide, and ethambutol pharmacokinetics in a cohort of tuberculosis patients. Antimicrob Agents Chemother. 2006;50(4):1170-7.

25. Requena-Mendez A, Davies G, Waterhouse D, Ardrey A, Jave O, Lopez-Romero SL, et al. Effects of dosage, comorbidities, and food on isoniazid pharmacokinetics in Peruvian tuberculosis patients. Antimicrob Agents Chemother. 2014;58(12):7164-70.

26. Taylor B, Smith PJ. Does AIDS impair the absorption of antituberculosis agents? Int J Tuberc Lung Dis. 1998;2(8):670-5.

27. Peloquin CA. Therapeutic drug monitoring in the treatment of tuberculosis. Drugs. 2002;62(15):2169-83.

28. Moher D, Liberati A, Tetzlaff J, Altman DG, PRISMA Group. Preferred reporting items for systematic reviews and meta-analyses: the PRISMA statement. J Clin Epidemiol. 2009;62(10):1006-12.

29. European Medicines Agency (EMA), Committee for Medicinal Product for Human Use. Guideline on the investigation on bioequivalence; 2010. http://www.ema.europa.eu/docs/en_GB/ document_library/Scientific_guideline/2010/01/WC50007003 9.pdf. Accessed 18 Dec 2017.

30. European Medicines Agency (EMA), Committee for Medicinal Product for Human Use. Guideline on the investigation of drug interactions; 2013. http://www.ema.europa.eu/docs/en_GB/docum
ent_library/Scientific_guideline/2012/07/WC500129606.pdf. Accessed 18 Dec 2017.

31. Kwara A, Enimil A, Gillani FS, Yang H, Sarfo AM, Dompreh A, et al. Pharmacokinetics of first-line antituberculosis drugs using WHO revised dosage in children with tuberculosis with and without HIV coinfection. J Pediatr Infect Dis Soc. 2016;5(4):356-65.

32. Antwi S, Yang H, Enimil A, Sarfo AM, Gillani FS, Ansong D, et al. Pharmacokinetics of the first-line antituberculosis drugs in Ghanaian children with tuberculosis with or without HIV coinfection. Antimicrob Agents Chemother. 2017;61(2):e01701-16.

33. Sahai J, Gallicano K, Swick L, Tailor S, Garber G, Seguin I, et al. Reduced plasma concentrations of antituberculosis drugs in patients with HIV infection. Ann Intern Med. 1997;127(4):289-93.

34. Peloquin CA, Nitta AT, Burman WJ, Brudney KF, MirandaMassari JR, McGuinness ME, et al. Low antituberculosis drug concentrations in patients with AIDS. Ann Pharmacother. 1996;30(9):919-25.

35. Jaruratanasirikul S. The pharmacokinetics of oral rifampicin in AIDS patients. J Med Assoc Thai. 1998;81(1):25-8.

36. Perlman DC, Segal Y, Rosenkranz S, Rainey PM, Peloquin CA, Remmel RP, ACTG 309 Team, et al. The clinical pharmacokinetics of pyrazinamide in HIV-infected persons with tuberculosis. Clin Infect Dis. 2004;38(4):556-64.

37. Perlman DC, Segal Y, Rosenkranz S, Rainey PM, Remmel RP, Salomon N, AIDS Clinical Trials Group 309 Team, et al. The clinical pharmacokinetics of rifampin and ethambutol in HIV-infected persons with tuberculosis. Clin Infect Dis. 2005;41(11):1638-47.

38. Ramachandran G, Kumar AK, Bhavani PK, Kannan T, Kumar SR, Gangadevi NP, et al. Pharmacokinetics of first-line antituberculosis drugs in HIV-infected children with tuberculosis treated with intermittent regimens in India. Antimicrob Agents Chemother. 2015;59(2):1162-7.

39. Choudhri SH, Hawken M, Gathua S, Minyiri GO, Watkins W, Sahai J, et al. Pharmacokinetics of antimycobacterial drugs in patients with tuberculosis, AIDS, and diarrhea. Clin Infect Dis. 1997;25(1):104-11.

40. Conte JE Jr, Golden JA, McQuitty M, Kipps J, Duncan S, McKenna E, et al. Effects of gender, AIDS, and acetylator status on intrapulmonary concentrations of isoniazid. Antimicrob Agents Chemother. 2002;46(8):2358-64.

41. Denti P, Jeremiah K, Chigutsa E, Faurholt-Jepsen D, PrayGod G, Range N, et al. Pharmacokinetics of isoniazid, pyrazinamide, and ethambutol in newly diagnosed pulmonary TB patients in Tanzania. PLoS One. 2015;10(10):e0141002.

42. Graham SM, Bell DJ, Nyirongo S, Hartkoorn R, Ward SA, Molyneux EM. Low levels of pyrazinamide and ethambutol in children with tuberculosis and impact of age, nutritional status, and human immunodeficiency virus infection. Antimicrob Agents Chemother. 2006;50(2):407-13.

43. Jeremiah K, Denti P, Chigutsa E, Faurholt-Jepsen D, PrayGod G, Range N, et al. Nutritional supplementation increases rifampin exposure among tuberculosis patients coinfected with HIV. Antimicrob Agents Chemother. 2014;58(6):3468-74.

44. Jonsson S, Davidse A, Wilkins J, Van der Walt JS, Simonsson US, Karlsson MO, et al. Population pharmacokinetics of ethambutol in South African tuberculosis patients. Antimicrob Agents Chemother. 2011;55(9):4230-7.

45. Bekker A, Schaaf HS, Draper HR, van der Laan L, Murray S, Wiesner L, et al. Pharmacokinetics of rifampin, isoniazid, pyrazinamide, and ethambutol in infants dosed according to revised WHO-recommended treatment guidelines. Antimicrob Agents Chemother. 2016;60(4):2171-9.

46. Conte JE, Golden JA, Kipps JE, Lin ET, Zurlinden E. Effect of sex and AIDS status on the plasma and intrapulmonary pharmacokinetics of rifampicin. Clin Pharmacokinet. 2004;43(6):395-404. 
47. Mukherjee A, Velpandian T, Singla M, Kanhiya K, Kabra SK, Lodha R. Pharmacokinetics of isoniazid, rifampicin, pyrazinamide and ethambutol in Indian children. BMC Infect Dis. 2015;15:126-015-0862-7.

48. van Oosterhout JJ, Dzinjalamala FK, Dimba A, Waterhouse D, Davies G, Zijlstra EE, et al. Pharmacokinetics of antituberculosis drugs in HIV-positive and HIV-negative adults in Malawi. Antimicrob Agents Chemother. 2015;59(10):6175-80.

49. Ramachandran G, Kumar AK, Kannan T, Bhavani PK, Kumar $\mathrm{SR}$, Gangadevi NP, et al. Low serum concentrations of rifampicin and pyrazinamide associated with poor treatment outcomes in children with tuberculosis related to HIV status. Pediatr Infect Dis J. 2016;35(5):530-4.

50. Rockwood N, Meintjes G, Chirehwa M, Wiesner L, McIlleron $\mathrm{H}$, Wilkinson RJ, et al. HIV-1 coinfection does not reduce exposure to rifampin, isoniazid, and pyrazinamide in south african tuberculosis outpatients. Antimicrob Agents Chemother. 2016;60(10):6050-9.

51. Schaaf HS, Willemse M, Cilliers K, Labadarios D, Maritz JS, Hussey GD, et al. Rifampin pharmacokinetics in children, with and without human immunodeficiency virus infection, hospitalized for the management of severe forms of tuberculosis. BMC Med. 2009;7:19.

52. Chideya S, Winston CA, Peloquin CA, Bradford WZ, Hopewell $\mathrm{PC}$, Wells $\mathrm{CD}$, et al. Isoniazid, rifampin, ethambutol, and pyrazinamide pharmacokinetics and treatment outcomes among a predominantly HIV-infected cohort of adults with tuberculosis from Botswana. Clin Infect Dis. 2009;48(12):1685-94

53. Schaaf HS, Parkin DP, Seifart HI, Werely CJ, Hesseling PB, van Helden PD, et al. Isoniazid pharmacokinetics in children treated for respiratory tuberculosis. Arch Dis Child. 2005;90(6):614-8.

54. Alsultan A, Peloquin CA. Therapeutic drug monitoring in the treatment of tuberculosis: an update. Drugs. 2014;74(8):839-54.

55. Boeree MJ, Diacon AH, Dawson R, Narunsky K, du Bois J, Venter A, PanACEA Consortium, et al. A dose-ranging trial to optimize the dose of rifampin in the treatment of tuberculosis. Am J Respir Crit Care Med. 2015;191(9):1058-65.

56. van Ingen J, Aarnoutse RE, Donald PR, Diacon AH, Dawson R, Plemper van Balen $\mathrm{G}$, et al. Why do we use $600 \mathrm{mg}$ of rifampicin in tuberculosis treatment? Clin Infect Dis. 2011;52(9):e194-9.

57. Peloquin CA, Jaresko GS, Yong CL, Keung AC, Bulpitt AE, Jelliffe RW. Population pharmacokinetic modeling of isoniazid, rifampin, and pyrazinamide. Antimicrob Agents Chemother. 1997;41(12):2670-9.

58. Alsultan A, Savic R, Dooley KE, Weiner M, Whitworth W, MacKenzie WR, Tuberculosis Trials Consortium, et al. Population pharmacokinetics of pyrazinamide in patients with tuberculosis. Antimicrob Agents Chemother. 2017;61(6):e02625-16.

59. Weiner M, Peloquin C, Burman W, Luo CC, Engle M, Prihoda $\mathrm{TJ}$, et al. Effects of tuberculosis, race, and human gene SLCO1B1 polymorphisms on rifampin concentrations. Antimicrob Agents Chemother. 2010;54(10):4192-200.

60. Sturkenboom MG, Mulder LW, de Jager A, van Altena R, Aarnoutse RE, de Lange WC, et al. Pharmacokinetic modeling and optimal sampling strategies for therapeutic drug monitoring of rifampin in patients with tuberculosis. Antimicrob Agents Chemother. 2015;59(8):4907-13.

61. Alffenaar JW. Dried blood spot analysis combined with limited sampling models can advance therapeutic drug monitoring of tuberculosis drugs. J Infect Dis. 2012;205(11):1765-6 (author reply 1766).

62. Zuur MA, Bolhuis MS, Anthony R, den Hertog A, van der Laan T, Wilffert B, et al. Current status and opportunities for therapeutic drug monitoring in the treatment of tuberculosis. Expert Opin Drug Metab Toxicol. 2016;12(5):509-21.

63. Semvua HH, Kibiki GS, Kisanga ER, Boeree MJ, Burger DM, Aarnoutse R. Pharmacological interactions between rifampicin and antiretroviral drugs: challenges and research priorities for resource-limited settings. Ther Drug Monit. 2015;37(1):22-32.

64. Regazzi M, Carvalho AC, Villani P, Matteelli A. Treatment optimization in patients co-infected with HIV and Mycobacterium tuberculosis infections: focus on drug-drug interactions with rifamycins. Clin Pharmacokinet. 2014;53(6):489-507.

65. Saktiawati AM, Sturkenboom MG, Stienstra Y, Subronto YW, Sumardi, Kosterink JG, et al. Impact of food on the pharmacokinetics of first-line anti-TB drugs in treatment-naive TB patients: a randomized cross-over trial. J Antimicrob Chemother. 2016;71(3):703-10.

66. Nahid P, Dorman SE, Alipanah N, Barry PM, Brozek JL, Cattamanchi A, et al. Official American Thoracic Society/Centers for Disease Control and Prevention/Infectious Diseases Society of America Clinical Practice Guidelines: treatment of drug-susceptible tuberculosis. Clin Infect Dis. 2016;63(7):e147-95.

67. Alffenaar JC, Tiberi S, Verbeeck RK, Heysell SK, Grobusch MP. therapeutic drug monitoring in tuberculosis: practical application for physicians. Clin Infect Dis. 2017;64(1):104-5.

68. Daskapan A, de Lange WC, Akkerman OW, Kosterink JG, van der Werf TS, Stienstra Y, et al. The role of therapeutic drug monitoring in individualised drug dosage and exposure measurement in tuberculosis and HIV co-infection. Eur Respir J. 2015;45(2):569-71.

69. Peloquin $\mathrm{C}$. The role of therapeutic drug monitoring in mycobacterial infections. Microbiol Spectr. 2017. https://doi.org/10.1128/ microbiolspec.TNMI7-0029-2016.

70. L'homme RF, Muro EP, Droste JA, Wolters LR, van EwijkBeneken Kolmer NW, Schimana W, et al. Therapeutic drug monitoring of nevirapine in resource-limited settings. Clin Infect Dis. 2008;47(10):1339-44.

71. Vu DH, Koster RA, Bolhuis MS, Greijdanus B, Altena RV, Nguyen DH, et al. Simultaneous determination of rifampicin, clarithromycin and their metabolites in dried blood spots using LC-MS/MS. Talanta. 2014;121:9-17.

72. Hiruy H, Rogers Z, Mbowane C, Adamson J, Ngotho L, Karim F, et al. Subtherapeutic concentrations of first-line anti-TB drugs in South African children treated according to current guidelines: the PHATISA study. J Antimicrob Chemother. 2015;70(4):1115-23.

73. Colangeli R, Jedrey H, Kim S, Connell R, Ma S, Chippada Venkata UD, DMID 01-009/Tuberculosis Trials Consortium Study 22 Teams, et al. Bacterial factors that predict relapse after tuberculosis therapy. N Engl J Med. 2018;379(9):823-33. 


\section{Affiliations}

\section{Alper Daskapan ${ }^{1}$. Lusiana R. Idrus ${ }^{2}$ - Maarten J. Postma ${ }^{2,3}$ - Bob Wilffert ${ }^{1,2} \cdot$ Jos G. W. Kosterink ${ }^{1,2}$. Ymkje Stienstra ${ }^{4}$. Daniel J. Touw ${ }^{1,5}$. Aase B. Andersen ${ }^{6}$. Adrie Bekker ${ }^{7}$. Paolo Denti ${ }^{8}$. Agibothu K. Hemanth Kumar ${ }^{9}$. \\ Kidola Jeremiah $^{10}$. Awewura Kwara ${ }^{11}$. Helen Mcllleron ${ }^{8}$. Graeme Meintjes ${ }^{12}$. Joep J. van Oosterhout ${ }^{13,14}$. Geetha Ramachandran ${ }^{9}$. Neesha Rockwood ${ }^{12,15}$ • Robert J. Wilkinson ${ }^{12,15,16}$. Tjip S. van der Werf ${ }^{4}$. Jan-Willem C. Alffenaar ${ }^{1}$}

1 Department of Clinical Pharmacy and Pharmacology, University of Groningen, University Medical Center Groningen, PO Box 30.001, 9700 RB Groningen, The Netherlands

2 Unit of PharmacoTherapy, -Epidemiology and -Economics, Groningen Research Institute of Pharmacy, University of Groningen, Groningen, The Netherlands

3 Department of Health Sciences, University of Groningen, University Medical Center Groningen, Groningen, The Netherlands

4 Department of Internal Medicine-Infectious Diseases, University of Groningen, University Medical Center Groningen, Groningen, The Netherlands

5 Unit of Pharmacokinetics, Toxicology and Targeting, Groningen Research Institute of Pharmacy, University of Groningen, Groningen, The Netherlands

6 Department of Infectious Diseases, Rigshospitalet, Copenhagen, Denmark

7 Department of Paediatrics and Child Health, University of Stellenbosch, Cape Town, South Africa
8 Division of Clinical Pharmacology, Department of Medicine, University of Cape Town, Cape Town, South Africa

9 National Institute for Research in Tuberculosis (ICMR), Chennai, India

10 National Institute for Medical Research, Mwanza Medical Research Centre, Mwanza, Tanzania

11 College of Medicine and Emerging Pathogens Institutes, University of Florida, Gainesville, FL, USA

12 Institute of Infectious Diseases and Molecular Medicine, Wellcome Centre for Infectious Diseases Research in Africa, University of Cape Town, Cape Town, South Africa

13 Dignitas International, Zomba, Malawi

14 Department of Medicine, College of Medicine, University of Malawi, Blantyre, Malawi

15 Department of Medicine, Imperial College London, London, UK

16 The Francis Crick Institute, London, UK 